\title{
Interleukin-2 in the treatment of unresectable or metastatic renal cell cancer: a systematic review and practice guideline
}

\author{
Sebastien Hotte, MD; ${ }^{*}$ Tricia Waldron, MSc; ${ }^{+}$Christina Canil MD; ${ }^{+}$ \\ Eric Winquist $M D^{\mathcal{S}}$ on behalf of the Cancer Care Ontario \\ Program in Evidence-based Care's Genitourinary Cancer \\ Disease Site Group
}

See related article on page 39

\begin{abstract}
Objective: We performed a systematic review of randomized controlled trials (RCTs) to assess the efficacy of interleukin-2 (IL-2) for the treatment of patients with unresectable or metastatic renal cell carcinoma (RCC).

Methods: We searched the literature to identify RCTs or meta-analyses of RCTs comparing treatment regimens with IL-2 to those without. Outcomes of interest included overall or progression-free survival, response rate, toxicity and quality of life.
\end{abstract}

Results: We identified 36 RCTs, and 6 met the eligibility criteria (1098 patients). We studied IL-2 alone and in combination with other agents, including interferon-alpha (IFN-a), 5-fluorouracil (5-FU), and 13-cis-retinoic acid or tamoxifen. No trials comparing high-dose IL-2 to non-IL-2 regimens were identified. A meta-analysis of 1-year mortality data from the 6 trials did not show a difference between IL-2-based regimens and non-IL-2 controls. Two of the 6 trials detected statistically significant longer survival with IL-2 combined with IFN-a and 5-FU. Of the 4 trials that assessed progression-free survival, 3 reported significantly longer progression-free intervals with IL-2-based regimens. Five trials reported response rates; pooling the rates from these trials gave an overall weighted response rate of $13.3 \%$ (range $9 \%-39 \%$ ) and $5.3 \%$ (range $0 \%-20 \%$ ) for IL-2-containing regimens and non-IL-2 regimens, respectively. IL-2-based regimens were more toxic than were non-IL-2 controls; the most frequently reported grade 3-4 toxicities were hypotension (range $6 \%-68 \%)$, fever $(2 \%-56 \%)$, nausea or vomiting or both $(6 \%-34 \%)$, diarrhea $(1 \%-28 \%)$ and cardiac toxicity $(11 \%-25 \%)$. None of the trials reported healthrelated quality-of-life data.

Conclusion: Non-high-dose IL-2 containing regimens do not provide superior treatment efficacy over non-IL-2-based regimens, with added toxicity, and therefore should not be used as standard treatment for patients with unresectable or metastatic RCC. High-dose IL-2 should only be used by experienced physicians in the context of a clinical trial or investigative setting.

CUAJ 2007;1(1):27-38

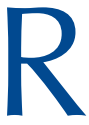
enal cell carcinomas (RCCs) account for $3 \%$ of all adult solid malignancies. ${ }^{1}$ In 2006, it was estimated that 4600 patients would be diagnosed with the disease in Canada. ${ }^{2}$ According to Bukowski, at the time of first diagnosis, $45 \%$ of patients will present with local- ized disease, 25\% will have locally advanced disease with lymph node or local organ involvement and the remaining 30\% will present with metastases. ${ }^{3}$ Patients with meta-static disease have a 5-year life expectancy of less than $10 \%$ and a median survival time of less than 12 months. However, survival can be quite variable, depending on several prognostic factors, including performance status, lactate dehydrogenase (LDH), hemoglobin and calcium levels, and the absence of prior nephrectomy. ${ }^{4}$

For patients presenting with inoperable or metastatic disease, cure is rarely possible, and treatment efforts often focus on effectively controlling symptoms and offering a chance at improved survival. Clinical trials of chemotherapy in the metastatic setting have shown RCCs to be resistant to currently available chemotherapeutic agents. ${ }^{5}$ Immunotherapy agents, however, have shown activity in RCC. Interleukin-2 (IL-2) has been evaluated extensively in the setting of advanced RCC. Various doses and modes of delivery have been studied, attempting to maximize efficacy and decrease the significant toxicities that can be associated with high-dose IL-2 therapy. Highdose IL-2 has been defined as IL-2 administered as an intravenous bolus of at least $600000 \mathrm{lU} / \mathrm{kg}$ every 8 hours, or a dose exceeding $65 \mathrm{mu} / \mathrm{m}^{2}$ daily. IL-2 at $18 \mathrm{mu} / \mathrm{m}^{2}$ days $1-4$ or 5 intravenously is not considered high-dose IL-2.

Since its approval by the United States Food and Drug Administration (FDA), highdose IL-2 has been used in patients with advanced RCC as a standard therapy at many comprehensive cancer centres in the US. This has likely contributed to the paucity of trials 
without an IL-2 arm. IL-2 was also approved by Health Canada in 2003, but its use has been very sporadic and limited. Because interferon-alpha (IFN-a) is associated with a real, if modest, survival benefit in the randomized setting 6 and because it is accessible to and tolerated by most RCC patients, it has become a de facto standard of care in Canada.

To clarify the role of IL-2 in the treatment of RCC and to develop appropriate recommendations for treatment, the Genitourinary Cancer Disease Site Group (GU DSG) of Cancer Care Ontario's Program in Evidence-based Care (PEBC) systematically reviewed evidence from randomized controlled trials (RCTs) of IL2 in patients with unresectable or metastatic RCC.

\section{Methods}

\section{Systematic review}

\section{Search strategy}

We searched the MEDLINE (1966-2005), EMBASE (1980-2005) and CancerLIT (1975-2002) databases for relevant papers, using disease-specific, treatmentspecific and design-specific medical subject headings and text words. In addition, we searched the Cochrane Library databases (2004, Issue 4), the conference proceedings of the American Society of Clinical Oncology (1995-2005) and the American Urological Association (1995-2005) for abstracts of relevant trials. We also searched the reference lists from eligible articles and relevant reviews for additional trials.

\section{Inclusion criteria}

Multiple regimen schedules, doses and types of immunotherapy have been studied in combination with and in comparison to IL-2, which makes it difficult to directly assess the potential benefits of IL-2. Therefore, we selected articles for inclusion in the systematic review if they were fully published reports or abstracts of RCTs or meta-analyses of RCTs comparing treatment regimens with IL-2 to regimens without IL-2 in patients with unresectable or metastatic RCC. Reports were required to provide data on at least one of the following outcomes: survival (i.e., overall, progression-free or time-toprogression), response rate, toxicity or quality of life. Reports that included nonRCC patients were eligible if the outcomes for RCC patients were analyzed separately. Relevant systematic reviews or evidence-based practice guidelines were also eligible. We excluded RCTs that compared either surgery or radiotherapy with IL-2 immunotherapy.

\section{Statistical methods}

\section{Objective response rate}

To estimate the overall effect of IL-2-based immunotherapy on response rate compared with non-IL-2 regimens, we pooled and weighted the response rates from individual RCTs according to the size of the treatment arms.

\section{Mortality at $l$ year}

Survival data were available for all 6 RCTs included in this review, either reported in the text or extractable from survival curves. To estimate the overall effect of IL-2-based immunotherapy on mortality, we pooled the data at a common time point (e.g., mortality at 1 year). Since time points before the median will generally ensure that there are sufficient data to be credible, a pooled median survival time (weighted by the size of the treatment arms) was calculated to determine an appropriate time for pooling. We performed the meta-analysis, using 1-year mortality data, because the pooled weighted median survival times were 19.4 months and 10.8 months for IL-2-containing arms and non-IL-2 arms, respectively. 
For 3-arm trials, we combined mortality data from each IL-2-containing arm and then entered these into the metaanalysis, so that each arm only contributed once to the meta-analysis.

We performed the meta-analysis with Review Manager 4.2, available through the Cochrane Collaboration. We used the random effects model as the more conservative estimate of treatment effect, ${ }^{8}$ and we expressed the results as relative risks (RRs) with 95\% confidence intervals (Cls). An RR less than 1.0 favours the experimental arm (i.e., IL-2-based immunotherapy) and an RR greater than 1.0 favours the control arm. We examined the metaanalysis results for heterogeneity by visual inspection of the forest plot and by calculating the Cochran $\mathrm{Q}$ test (using a planned cut-off for significance of $p<0.05$ ) and $\mathrm{I}^{2}$ statistics (values of $25 \%, 50 \%$ and $75 \%$ indicate low, moderate and high degrees of heterogeneity). ${ }^{9}$

\section{Clinical practice guideline and external review}

On the basis of the evidence in the systematic review, draft recommendations were developed by the GU DSG through consensus to create a clinical practice guideline. The clinical practice guideline is intended to promote evidence-based practice in Ontario. As part of the PEBC's practice guideline development cycle, all draft recommendations are sent to Ontario practitioners for external review. The external review is a mailed survey consisting of items that address the quality of the draft recommendations and whether the draft recommendations should serve as a clinical practice guideline. We sent the surveys to a random sample $(n=92)$ of Ontario practitioners, (medical oncologists and urologists) with expertise in treating genitourinary cancers. We sent follow-up reminders at 2 weeks and 4 weeks. The GU DSG reviewed the survey results and considered the comments written by the practitioners before finalizing the clinical practice guideline.

\section{Results}

\section{Literature search results}

We identified 36 unique RCTs of IL-2 through the literature search; only 7 of those met the eligibility criteria. ${ }^{10-16} \mathrm{We}$ excluded 1 of the 7 eligible trials because most of the patients (58\%) were lost to follow-up. ${ }^{11}$ All 6 trials were published as full reports in journals. We also identified 3 systematic reviews with metaanalyses ${ }^{6,17,18}$; of these, 2 were excluded because they pooled data from both randomized and nonrandomized clinical trials. ${ }^{17,18}$ We identified no evidence-based guidelines.

\section{Previous systematic review with meta-analysis}

In 2000, Coppin and colleagues published a Cochrane systematic review on immunotherapy for inoperable or metastatic RCC. In 2004, the review was updated to cover the literature through to December 2003. ${ }^{6}$ The review examined the efficacy of a range of immunotherapies, including IL-2. Eligible reports were RCTs examining any immunotherapy in at least 1 trial arm that reported results on mortality or remission by treatment arm. Metaanalyses were performed for both outcomes, using data available from published trial reports, and treatment comparisons were specified a priori. Only 3 comparisons evaluated in the Cochrane review (as subgroup analyses) met our review criteria (i.e., compared IL-2-based regimens to nonIL-2 regimens) and include the following: 1) high-dose IL-2 versus non-IL-2 controls, 2) IL-2 plus IFN-a versus IFN-a alone, and 3) IL-2-based regimens versus IFN-a alone. We did not identify any evidence-based guidelines that addressed comparison 1 , and we identified 2 trials that addressed comparisons 2 and $3 .^{13,15}$ The meta-analysis results are based on subgroup analyses and are limited by the inclusion of only 2 trials and a small number of patients (204 to 329 patients, depending on the comparison and outcome). Results from the meta-analyses 
showed no differences between IL-2-based regimens and non-IL-2 regimens in both 1year mortality and remission. IL-2 combined with IFN-a was associated with a statistically significant improvement in remission, compared with IFN-a alone, but that did not translate into an improvement in survival at 1 year. From their review of 22 trials, Coppin and colleagues ${ }^{6}$ concluded that, because high-dose IL-2 has not been compared with another therapy, its possible superiority must be based on the results of phase II studies, which were not reviewed in the Cochrane overview. The authors also concluded that modified or reduced schedules of IL-2 should not be recommended outside of clinical trials until studies with adequate patient numbers show greater efficacy than IFN-a or equivalence with less toxicity.

\section{Randomized controlled trials}

\section{Trial characteristics}

The 6 trials that form the basis of this review were published between 1996 and 2004 (Table 1). There were three 3 -arm trials ${ }^{10,13,15}$ and three 2-arm trials. ${ }^{12,14,16}$ Across these trials, 1098 eligible patients were randomized, with patient accruals per trial ranging from 60 to 425. None of the trials were placebocontrolled. All of the trials assessed IL-2 in combination with other agents, and 2 of the 3-arm trials also included a single-agent IL-2 arm. ${ }^{13,15}$ IL-2 was studied in combination with IFN-a (2a or $2 b$ ) in each trial, either alone $e^{13,15,16}$ or with 5-fluorouracil (5-FU) ${ }^{10,12}$ or 5 -FU and 13 -cis retinoic acid $(13 \mathrm{cRA})^{10}$ or tamoxifen. ${ }^{14}$ The doses and route of administration of IL-2 differed significantly between trials. Four trials evaluated IL-2 given subcutaneously ( 2 at a low dose of 2.4 to $4.8 \mathrm{mu} / \mathrm{m}^{2}$ ), and 2 trials evaluated IL-2 administered intravenously at a dose of $18 \mathrm{mu} / \mathrm{m}^{2}$. The median age of patients ranged from 57 to 62 years, and most patients were men (range 59\%-82\%) with good performance status (i.e., Karnofsky $>80 \%$, World

Table 1: IL-2 regimens versus non-IL-2 in unresectable or metastastic RCC: trial descriptions

\begin{tabular}{|c|c|c|}
\hline Trial & Treatment arms (dose and route) & $\begin{array}{l}\text { No. patients } \\
\text { randomized }\end{array}$ \\
\hline $\begin{array}{l}\text { Atzpodien et al, } \\
2004^{10}\end{array}$ & $\begin{array}{l}\text { A: IFN-a2a }\left(5 \rightarrow 10 \mathrm{mu} / \mathrm{m}^{2} \mathrm{sc}\right), \mathrm{IL}-2\left(10 \rightarrow 5 \mathrm{mu} / \mathrm{m}^{2}\right. \\
\mathrm{sc}), 5-F U\left(1000 \mathrm{mg} / \mathrm{m}^{2} \mathrm{IV}\right) \\
\text { B: IFN-a2a }\left(5 \rightarrow 10 \mathrm{mu} / \mathrm{m}^{2} \mathrm{sc}\right), \mathrm{IL}-2\left(10 \rightarrow 5 \mathrm{mu} / \mathrm{m}^{2}\right. \\
\text { sc), 5-FU }\left(1000 \mathrm{mg} / \mathrm{m}^{2} \mathrm{IV}\right), 13 c R A(20 \mathrm{mg} \text { orally) } \\
\text { C: IFN-a2a }\left(5 \rightarrow 10 \mathrm{mu} / \mathrm{m}^{2} \mathrm{sc}\right), \mathrm{VBL}\left(6 \mathrm{mg} / \mathrm{m}^{2} \mathrm{IV}\right)\end{array}$ & 341 \\
\hline $\begin{array}{l}\text { Atzpodien et al, } \\
2001^{12}\end{array}$ & $\begin{array}{l}\text { A: IL-2 }\left(10 \rightarrow 5 \mathrm{mu} / \mathrm{m}^{2} \mathrm{sc}\right), \text { IFN-a }\left(5 \rightarrow 10 \mathrm{mu} / \mathrm{m}^{2} \mathrm{sc}\right) \text {, } \\
5 \text {-FU }\left(1000 \mathrm{mg} / \mathrm{m}^{2} \mathrm{IV}\right) \\
\text { B: Tamoxifen ( } 80 \mathrm{mg} \text { orally) }\end{array}$ & 78 \\
\hline $\begin{array}{l}\text { Boccardo et al, } \\
1998^{13}\end{array}$ & $\begin{array}{l}\text { A: IL-2 }\left(18 \mathrm{mu} / \mathrm{m}^{2} \mathrm{IV}\right) \\
\text { B: IL-2 (18 } \mathrm{mu} / \mathrm{m}^{2} \text { IV), IFN-a2a }\left(6 \mathrm{mu} / \mathrm{m}^{2} \mathrm{IM}\right) \\
\text { C: IFN-a2a }\left(6 \mathrm{mu} / \mathrm{m}^{2} \text { IM) }\right.\end{array}$ & 66 \\
\hline $\begin{array}{l}\text { Henriksson et al, } \\
1998^{14}\end{array}$ & $\begin{array}{l}\text { A: IL-2 }\left(4.8 \rightarrow 2.4 \mathrm{mu} / \mathrm{m}^{2} \mathrm{sc}\right), \mathrm{IFN}-\mathrm{a}\left(3 \rightarrow 6 \mathrm{mu} / \mathrm{m}^{2} \mathrm{sc}\right) \\
\text { tamoxifen }(40 \mathrm{mg} \text { orally) } \\
\text { B: Tamoxifen ( } 40 \mathrm{mg} \text { orally) }\end{array}$ & 128 \\
\hline Negrier et al, $1998^{15}$ & $\begin{array}{l}\text { A: IL-2 (18 mu/m } \mathrm{m}^{2} \text { IV) } \\
\text { B: IL-2 (18 } \mathrm{mu} / \mathrm{m}^{2} \text { IV), IFN-a2a }\left(6 \mathrm{mu} / \mathrm{m}^{2} \mathrm{sc}\right) \\
\text { C: IFN-a2a }\left(18 \mathrm{mu} / \mathrm{m}^{2} \mathrm{sc}\right)\end{array}$ & 425 \\
\hline Lummen et al, $1996^{16}$ & $\begin{array}{l}\text { A: IL-2 }\left(4.8 \rightarrow 2.4 \mathrm{mu} / \mathrm{m}^{2} \mathrm{sc}\right) \text { IFN-a2b }\left(3 \rightarrow 6 \mathrm{mu} / \mathrm{m}^{2}\right. \\
\mathrm{sc}) \\
\text { B: IFN- } \gamma(200 \mu \mathrm{g} \mathrm{sc})\end{array}$ & 60 \\
\hline
\end{tabular}


Health Organization or Eastern Cooperative Oncology Group $\leq 2$ ). The median follow-up of patients across the 6 trials was 22 months (range 11-39 mo).

We reviewed data indicative of trial quality, including methods of randomization and allocation concealment, adequacy of the trial arm description, whether analyses included all randomized patients, and completeness of follow-up for each trial. Four of the 6 trials reported the method of randomization used. ${ }^{10,12,14,15}$ The method of allocation concealment was not reported in any of the trials, and none of the trials were blinded. All of the trials presented baseline demographic and clinical character- istics for treatment and control arms, and 4 stated that randomization achieved balance in the distribution of those characteristics between arms..$^{10,12,13,15}$ Five trials reported the percentage of patients receiving intended treatment and withdrawing from the study, ${ }^{10,12-15}$ and all employed an intent-to-treat approach to statistical analyses. A crossover trial design was used in 3 trials $^{10,12,15}$; in each of those, patients crossed over to alternative therapy upon disease progression, after $8^{10,12}$ or 10 weeks $^{15}$ of assigned treatment.

\section{Outcomes}

We summarized the results of the 6

Table 2: IL-2 regimens versus non-IL-2 regimens in unresectable or metastatic RCC: trial outcomes

\begin{tabular}{|c|c|c|c|c|c|c|c|c|c|c|c|}
\hline \multirow[b]{2}{*}{ Trial } & \multirow[b]{2}{*}{$\begin{array}{l}\text { Treatment arms } \\
\text { (no. of patients) }\end{array}$} & \multicolumn{4}{|c|}{ Objective response rate, \% } & \multicolumn{4}{|c|}{ Survival } & \multicolumn{2}{|c|}{$\begin{array}{l}\text { Progression-free } \\
\text { survival }\end{array}$} \\
\hline & & OR & CR & PR & $\begin{array}{c}p \\
\text { value* }\end{array}$ & $\begin{array}{l}\text { Median, } \\
\text { mo }\end{array}$ & $\begin{array}{c}p \\
\text { value* }\end{array}$ & $\begin{array}{l}1- \\
\text { year, } \\
\%\end{array}$ & $\begin{array}{c}5- \\
\text { year, } \\
\%\end{array}$ & $\begin{array}{l}\text { Median, } \\
\text { mo }\end{array}$ & $\begin{array}{c}p \\
\text { value* }\end{array}$ \\
\hline \multirow[t]{3}{*}{$\begin{array}{l}\text { Atzpodien } \\
\text { et al, } 2004^{10}\end{array}$} & $\begin{array}{l}\text { A: IFN-a2a/IL-2/ } \\
\text { 5-FU (132) }\end{array}$ & 31 & 5 & 26 & \multirow{3}{*}{$\begin{array}{c}n s \\
A v . B \\
C\end{array}$} & 25 & $\begin{array}{c}0.04 \\
\text { A v. C }\end{array}$ & $76.5 \dagger$ & $16.1+$ & 6 & \multirow{3}{*}{$\begin{array}{l}0.025 \\
\text { B v. C }\end{array}$} \\
\hline & $\begin{array}{l}\text { B: IFN-a2a/IL-2/ } \\
\text { 5-FU/13cRA (146) }\end{array}$ & 26 & 8 & 18 & & 27 & $\begin{array}{c}0.02 \\
\text { B v. C }\end{array}$ & $70.5+$ & $22.3+$ & 7 & \\
\hline & $\begin{array}{l}\text { C: IFN-a2a/VBL } \\
\text { (63) }\end{array}$ & 20 & 6 & 14 & & 16 & & $58.9+$ & $19.6+$ & 5 & \\
\hline \multirow[t]{2}{*}{$\begin{array}{l}\text { Atzpodien } \\
\text { et al, } 2001^{12}\end{array}$} & $\begin{array}{l}\text { A: IL-2/IFN-a/5-FU } \\
\text { (41) }\end{array}$ & 39 & 17 & 22 & \multirow[t]{2}{*}{$\mathrm{nr}$} & 24 & \multirow[t]{2}{*}{0.03} & $80+$ & 24.8 & 7 & \multirow[t]{2}{*}{$<0.0001$} \\
\hline & B: Tamoxifen (37) & 0 & 0 & 0 & & 13 & & $52 \dagger$ & 13.5 & 0 & \\
\hline \multirow{3}{*}{$\begin{array}{l}\text { Boccardo } \\
\text { et al, } 1998^{13}\end{array}$} & A: IL-2(22) & 23 & 9 & 14 & \multirow{3}{*}{$\mathrm{nr}$} & $28.3+$ & \multirow{3}{*}{$\mathrm{nr}$} & $70+$ & \multirow{3}{*}{ na } & $9.6+$ & \multirow{3}{*}{$\mathrm{nr}$} \\
\hline & B: IL-2/IFN-a2a(22) & 9 & 4.5 & 4.5 & & $13.3+$ & & $56.5+$ & & $5.1+$ & \\
\hline & C: IFN-a2a(22) & 9 & 0 & 9 & & $17.6+$ & & $76+$ & & $6.4 \dagger$ & \\
\hline \multirow[t]{2}{*}{$\begin{array}{l}\text { Henriksson } \\
\text { et al, } 1998^{14}\end{array}$} & $\begin{array}{l}\text { A: IL-2/IFN-a/ } \\
\text { tamoxifen (65) }\end{array}$ & $\mathrm{nr}$ & 7.7 & NR & \multirow[t]{2}{*}{$\mathrm{nr}$} & 11.8 & $\mathrm{~ns}$ & 40 & \multirow[t]{2}{*}{ na } & \multirow[t]{2}{*}{$\mathrm{nr}$} & \multirow{7}{*}{$\begin{array}{c}0.01 \\
B \vee . A \\
C\end{array}$} \\
\hline & B: Tamoxifen (63) & & 3 & & & 13.3 & & 48 & & & \\
\hline \multirow[t]{3}{*}{$\begin{array}{l}\text { Negrier } \\
\text { et al, } 1998^{15}\end{array}$} & A: IL-2 (138) & $6.5 \ddagger$ & $1.4 \ddagger$ & $5.1 \neq$ & \multirow{3}{*}{$\begin{array}{c}<0.01 \\
\text { B v. A } \\
\text { C }\end{array}$} & 12 & \multirow{3}{*}{$\begin{array}{c}\mathrm{ns} \\
\mathrm{B} \vee \mathrm{A} \text {, } \\
\mathrm{C}\end{array}$} & $56.8 \dagger$ & & $\begin{array}{c}15 \text { (PFS } \\
1 \mathrm{yr})\end{array}$ & \\
\hline & $\begin{array}{l}\text { B: IL-2/IFN-a2a } \\
(140)\end{array}$ & $18.6 \neq$ & $<1 \neq$ & $18 \ddagger$ & & 17 & & $60.2 \dagger$ & na & $\begin{array}{c}20 \text { (PFS } \\
1 \mathrm{yr})\end{array}$ & \\
\hline & C: IFN-a2a (147) & $7.5 \neq$ & $0 \ddagger$ & $7.5 \ddagger$ & & 13 & & $66.3+$ & & $\begin{array}{c}12 \text { (PFS } \\
1 \mathrm{yr})\end{array}$ & \\
\hline \multirow[t]{2}{*}{$\begin{array}{l}\text { Lummen } \\
\text { et al, } 1996^{16}\end{array}$} & $\begin{array}{l}\text { A: IL-2/IFN-a2b } \\
(30)\end{array}$ & 23 & 10 & 13 & \multirow{2}{*}{0.01} & 12 & \multirow[t]{2}{*}{0.49} & $50+$ & \multirow[t]{2}{*}{ na } & \multirow[t]{2}{*}{$\mathrm{nr}$} & \\
\hline & B: IFN- $\gamma(30)$ & 0 & 0 & 0 & & 13 & & $54+$ & & & \\
\hline \multicolumn{12}{|c|}{ 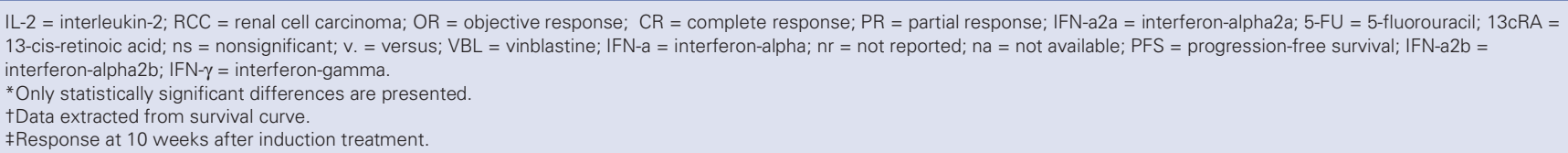 } \\
\hline
\end{tabular}


RCTs by outcome in Table 2 and Table 3 . Objective response rate was designated the primary outcome in 4 trials $\mathrm{s}^{10,12,13,15}$ and was reported in 5 trials ${ }^{10,12,13,15,16}$ (Table 2). Overall survival and progression-free survival data were reported in 6 and 4 trials, $10,12,13,15$ respectively (Table 2). All 6 trials reported toxicity data ${ }^{10,12-16}$ (Table 3). Quality-of-life data were not reported in any of the trial reports.

\section{Objective response rate}

Of the 5 trials reporting objective response rates, 3 were 3 -arm trials, providing 2 comparisons of IL-2-based immunotherapy, for a total of 8 comparisons among the 5 trials (Table 2). Only 3 trials provided statistical comparisons of those data ${ }^{10,15,16}$; 2 trials ( 3 comparisons) reported statistically significant higher response rates with IL-2. ${ }^{15,16}$

Table 3: IL-2 regimens versus non-IL-2 regimens in unresectable or metastatic RCC: reported toxicity

\begin{tabular}{|c|c|c|c|}
\hline Trial & Treatment arms & Reported grade $3-4$ toxicity (\% of patients) & $\begin{array}{l}\text { Reported no. } \\
\text { of toxic deaths }\end{array}$ \\
\hline \multirow[t]{3}{*}{$\begin{array}{l}\text { Atzpodien et al, } \\
2004^{10}\end{array}$} & A: IFN-a/IL-2/5-FU & $\begin{array}{l}\text { Fever (3), chills (3), malaise (3), diarrhea (3), respiratory distress (3), skin } \\
\text { or allergies (3), hemoglobin levels (4) }\end{array}$ & 0 \\
\hline & $\begin{array}{l}\text { B: IFN-a/IL-2/5- } \\
\text { FU/13cRA }\end{array}$ & $\begin{array}{l}\text { Fever (3), chills (6), malaise (18), nausea or vomiting (6), anorexia (21), } \\
\text { diarrhea (3), respiratory distress (3), skin or allergies (2), mucositis (3), } \\
\text { hypotension (6), alopecia (3), arrhythmias (3), paresthesias (3), leucocyte } \\
\text { count (3) }\end{array}$ & 0 \\
\hline & C: IFN-a/NBL & Malaise (11), anorexia (26), CNS symptoms or disorientation (11) & 0 \\
\hline \multirow{2}{*}{$\begin{array}{l}\text { Atzpodien et al, } \\
2001^{12}\end{array}$} & A: IL-2/IFN-a/5-FU & Fever (2), chills (3), malaise (7), diarrhea (1), dyspnea (1) & 0 \\
\hline & B: Tamoxifen & None reported & 0 \\
\hline \multirow[t]{3}{*}{$\begin{array}{l}\text { Boccardo et al, } \\
1998^{13}\end{array}$} & A: IL-2 & $\begin{array}{l}\text { Oliguria (17), hypotension (28), skin rash (11), fever (11), creatinemia (6), } \\
\text { neurological toxicity (11), cardiac toxicity (11), diarrhea (17), } \\
\text { nausea/vomiting (6) }\end{array}$ & $\mathrm{nr}$ \\
\hline & $\mathrm{B}:$ IL-2/IFN-a2a & $\begin{array}{l}\text { Oliguria (19), hypotension (38), skin rash (6), fever (25), cardiac toxicity } \\
\text { (25), diarrhea (19), nausea/vomiting (19), thrombocytopenia (6) }\end{array}$ & $\mathrm{nr}$ \\
\hline & C: IFN-a2a & Oliguria (6), hypotension (6), asthenia (11) & $\mathrm{nr}$ \\
\hline \multirow[t]{2}{*}{$\begin{array}{l}\text { Henriksson et al, } \\
1998^{14}\end{array}$} & $\begin{array}{l}\text { A: IL-2/IFN-a/ } \\
\text { tamoxifen }\end{array}$ & $\begin{array}{l}\text { Fatigue (58), anorexia (22), nausea (22), fever (12), diarrhea (8), myalgia } \\
\text { (18), pulmonary symptoms (14), infection (3), cutaneous }(<2) \text {, headache } \\
\text { (3), oral symptoms }(<2) \text {, CNS symptoms }(<2)\end{array}$ & $\mathrm{nr}$ \\
\hline & B: Tamoxifen & $\begin{array}{l}\text { Fatigue (30), anorexia (11), nausea (8), diarrhea (3), myalgia (22), } \\
\text { pulmonary symptoms (17), oral symptoms }(<2) \text {, CNS symptoms }(<3)\end{array}$ & $\mathrm{nr}$ \\
\hline \multirow[t]{3}{*}{$\begin{array}{l}\text { Negrier et al, } \\
1998^{15}\end{array}$} & A: IL-2 & $\begin{array}{l}\text { Hypotension (68), fever (43), performance status impairment (36), } \\
\text { nausea/vomiting (34), diarrhea (28), anemia (17), pulmonary symptoms } \\
\text { (16), renal symptoms (15), neurological symptoms (12), increased AST or } \\
\text { ALT (11), cutaneous signs (10), cardiac signs (12), infection (8), } \\
\text { thrombocytopenia (4), increased creatinine (4), weight loss }(2) \text {, } \\
\text { leucopenia }(<1) \text {, hyperbilirubinemia }(<1)\end{array}$ & $\mathrm{nr}$ \\
\hline & $\mathrm{B}:$ IL-2/IFN-a2a & $\begin{array}{l}\text { Hypotension (67), fever (56), performance status impairment (38), } \\
\text { nausea/vomiting (31), diarrhea (25) anemia (16), pulmonary symptoms } \\
\text { (15), renal symptoms (16) neurological symptoms (14), increased AST or } \\
\text { ALT (11), cutaneous signs (14), cardiac signs (6), infection (9), } \\
\text { thrombocytopenia (7), increased creatinine (5), weight loss (1), } \\
\text { leucopenia (2), hyperbilirubinemia (2) }\end{array}$ & $\mathrm{nr}$ \\
\hline & C: IFN-a2a & $\begin{array}{l}\text { Hypotension }(<1) \text {, fever }(5) \text {, performance status impairment (16), } \\
\text { nausea/vomiting (5), diarrhea }(<1) \text {, anemia }(6) \text {, pulmonary symptoms (3), } \\
\text { neurological symptoms }(1) \text {, increased AST or ALT (3), cardiac signs }(<1) \text {, } \\
\text { infection }(<1) \text {, weight loss }(4) \text {, leucopenia }(<1)\end{array}$ & $\mathrm{nr}$ \\
\hline \multirow{2}{*}{$\begin{array}{l}\text { Lummen et al, } \\
1996^{16}\end{array}$} & A: IL-2, IFN-a2b & $\mathrm{nr}$ & $\mathrm{nr}$ \\
\hline & $\mathrm{B}: \mathrm{IFN}-\gamma$ & $\mathrm{nr}$ & $\mathrm{nr}$ \\
\hline
\end{tabular}


Negrier and colleagues ${ }^{15}$ reported an objective response rate of $18.6 \%$ with combination IL-2 and IFN-a2a versus $7.5 \%(p<0.01)$ and $6.5 \%(p<0.01)$ with single-agent IFN-a2a and single-agent IL2 , respectively. Statistical findings were not reported for the comparison of IL-2 and IFN-a2a. Lummen and colleagues ${ }^{16}$ also reported a higher objective response rate with combination IL-2 and IFN-a2b over IFN-gamma ( $23 \%$ v. $0 \%, p=0.01)$. In the 2 trials for which statistical comparisons were not provided, the trial by Atzpodien and colleagues ${ }^{12}$ showed response rates that favoured IL-2-based therapy, compared with tamoxifen, and the trial by Boccardo and others ${ }^{13}$ reported a better response rate with single-agent IL-2 versus both single-agent IFN-a2a and combined IFN-a2a and IL2 . When the objective response rates from the 5 trials were combined, the overall weighted objective response rates for IL-2-based regimens versus regimens that were not IL-2 based were $13.3 \%$ (range $9 \%-39 \%$ ) versus $5.3 \%$ (range $0 \%-20 \%)(p \leq 0.001)$.

\section{Survival}

All 6 trials reported survival data $(9 \mathrm{com}-$ parisons) (Table 2). Median survival data were reported in 4 trials $\mathrm{s}^{10,12,14,15}$ and extracted from survival curves in 2 trials. ${ }^{13,16}$ Five of the 6 trials provided statistical comparisons for median survival times between trial arms. ${ }^{10,12,14-16}$ Of those, 2 reported statistically significant survival improvements with IL-2-based immunotherapy. ${ }^{10,12}$ In the trial by Atzpodien and colleagues, ${ }^{10}$ median survival was longer for patients treated with a combination of IL-2 and IFN-a with either 5 -FU (25 mo; $p=0.04)$ or 13cRA (27 mo; $p=0.02$ ), compared with patients receiving combined treatment with IFN-a2a and vinblastine $(16 \mathrm{mo})$. In the earlier trial by Atzpodien and colleagues, ${ }^{12}$ a statistically significant longer median survival was observed with IL-2 combined with IFN-a and 5-FU over tamoxifen (24 v. 13 mo; $p=0.03$ ).
One-year mortality data were reported in one trial ${ }^{14}$ and were extracted from survival curves in 5 trials $^{10,12-15}$ (Table 2). When the data were pooled in a metaanalysis, no statistically significant difference was observed between IL-2-based regimens and non-IL-2 controls ( $\mathrm{RR}=0.94$; 95\% Cl, 0.67-1.30; $p=0.69$ ) (Fig. 1). Statistically significant heterogeneity was detected across the 6 trials $\left(p=0.003, \mathrm{I}^{2}=\right.$ $71.7 \%$ ) and was therefore explored through a sensitivity analysis. Visual inspection of the meta-analysis figure clearly identified the 2 trials by Atzpodien and others ${ }^{10,12}$ as the likely source of heterogeneity. Both trials evaluated immunochemotherapy (IL-2-based regimens with 5-FU), and each detected a statistically significant survival improvement with those regimens over control therapy. When both trials were removed from the metaanalysis, the overall result remained the same $(R R=1.20 ; 95 \% \mathrm{Cl}, 1.00-1.44$; $p=0.06)$, but heterogeneity was no longer statistically significant $(p=0.81$, $\mathrm{I}^{2}=0 \%$ ). The subgroup of the 2 trials by Atzpodien and others ${ }^{10,12}$ showed a statistically significant reduction in 1-year mortality with immunochemotherapy $(\mathrm{RR}=0.56 ; 95 \% \mathrm{Cl}, 0.38-0.82 ; p=0.003)$.

\section{Disease progression}

Of the 6 trials, 4 assessed progression-free survival ${ }^{10,12,13,15}$ (Table 3). Three of the trials reported statistical comparisons, 10,12,15 and each of those detected longer, statistically significant progression-free intervals with IL-2-based regimens over non-IL-2 controls. In the largest of those trials, ${ }^{15}$ median progression-free survival at 1 year was significantly longer for patients treated with IL-2 combined with IFN-a2a (20 mo), compared with singleagent IL-2 (15 mo; $p=0.01)$ and singleagent IFN-a2a (12 mo; $p=0.01)$.

\section{Toxicity}

All 6 trials reported on toxicity; however, grade 3 or 4 toxicity data were described 
Hotte et al

in 5 of the 6 trials $^{10,12-15}$ (Table 3). Overall, IL-2-containing regimens appeared more toxic than did non-IL-2 regimens but were described as moderately to well tolerated by most patients in most of the trials. In 2 trials, IL-2-based immunotherapy was administered in an outpatient setting..$^{10,12}$ Most toxicities were graded as 1 or 2 (data not shown), but grade 3 or 4 toxicity was observed in a substantial number of patients. The most common grade 3 or 4 toxicities associated with IL-2-based treatment were fever (range $2 \%-56 \%$ ), chills $(3 \%-6 \%)$, malaise $(3 \%-18 \%)$, anorexia $(11 \%-22 \%)$, oliguria $(6 \%-19 \%)$, nausea or vomiting or both $(6 \%-34 \%)$, diarrhea $(1 \%-28 \%)$, skin rash or allergies $(3 \%-11 \%)$, hypotension $(6 \%-68 \%)$, pulmonary distress $(3 \%-16 \%)$, and central nervous system $(<2 \%-14 \%)$ and cardiac toxicity $(11 \%-25 \%)$. Only 2 trials reported on toxicity-related dose reductions or treatment discontinuations. Dose reductions occurred in $7 \%$ of patients treated with combination IL-2/IFN-a/5-FU, compared with none with tamoxifen. ${ }^{12}$ The toxicity of combination IL-2-IFN-a and 5FU and IL-2/IFN-a/5-FU/13cRA caused treatment discontinuations in $4 \%$ and $6 \%$ of patients, respectively, compared with $8 \%$ in patients treated with IFN-a combined with vinblastine. ${ }^{10}$ No toxic deaths were reported in 2 trials reporting those data. ${ }^{10,12}$

\section{Results of external review}

Based on the evidence contained in the systematic review, the GU DSG developed a clinical practice guideline and circulated it for external review. Of the 92 surveys sent, 39 were returned $(42 \%$ response rate). Of the practitioners who responded, $67 \%$ indicated that the report was relevant to their clinical practice and completed the survey. The key survey results include agreement with the summary and interpretation of evidence (96\%); agreement with the recommendations (88\%); and approval of the recommendations as a clinical practice guideline $(77 \%)$.

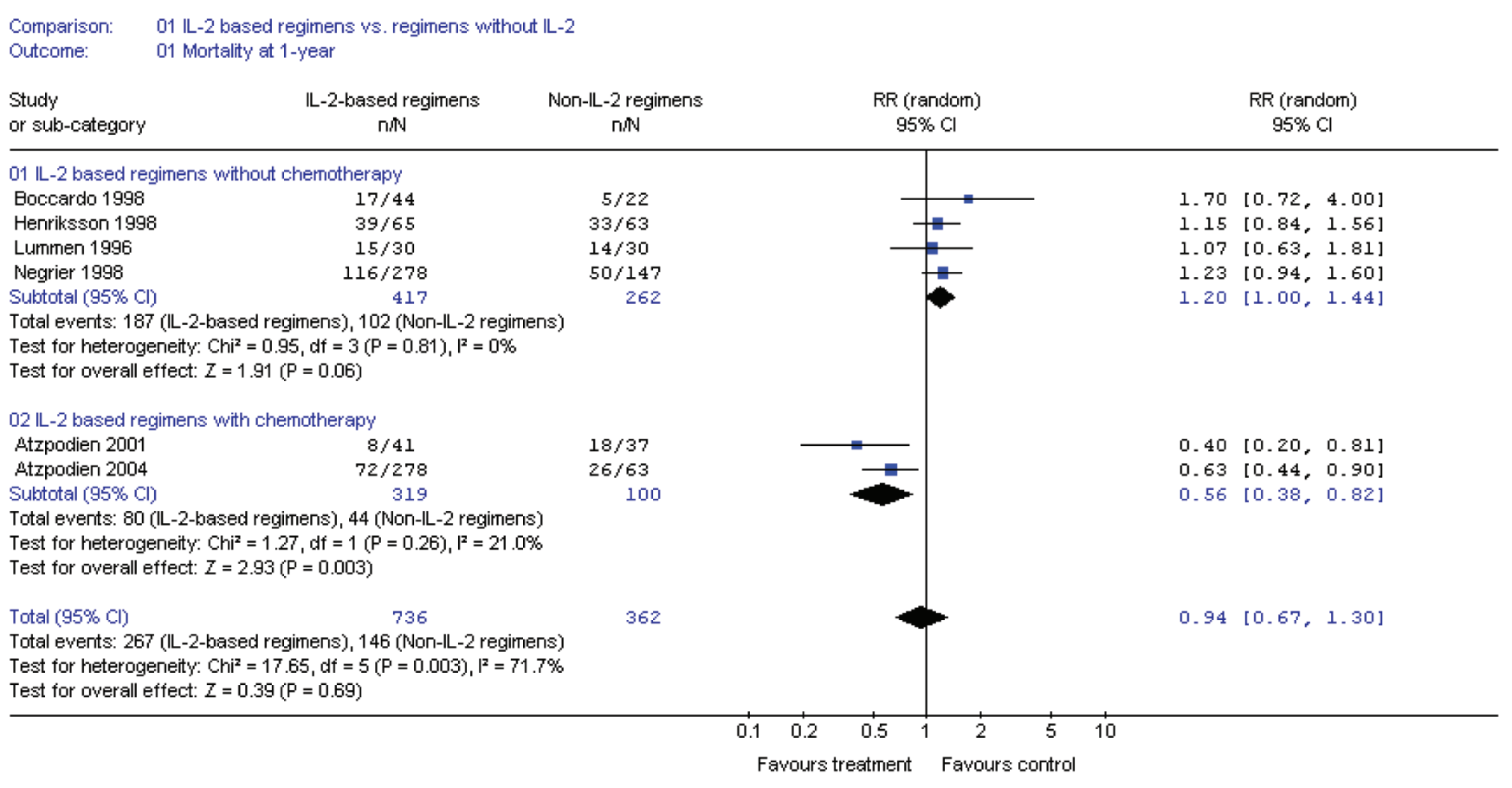

Fig. 1: IL-2 regimens versus non-IL-2 regimens in unresectable or metastatic RCC: meta-analysis of 1-year mortality data. 
Five respondents (19\%) provided written comments. One respondent commented that phase II trials should be included in the review because of the limited evidence and the small number of patients included in the randomized trials of IL-2. Another respondent commented that some patients benefit from highdose IL-2, but prior identification of these patients is not possible at this time. Therefore, patients need to be entered into trials of IL-2, but this agent is not available for study in Ontario. The respondent further commented that the field of study is changing with the use of angiogenesis inhibitors (i.e., sorafenib and sunitinib).

\section{Discussion}

Of the 34 trials in this review, only 6 met the inclusion criteria. The main reason for the large attrition proportion is that most trials did not contain an arm without IL-2. The 6 eligible RCTs evaluated IL-2 in modified doses, either intravenously or subcutaneously, in comparison to a variety of regimens. None evaluated high-dose IL-2, because IL2 at $18 \mathrm{mu} / \mathrm{m}^{2}$ days $1-4$ or 5 intravenously is not considered high-dose Il-2. Response rates were higher in patients receiving IL-2-based regimens (range, 6.5\%-39\%), compared with non-IL-2 controls $(0 \%-20 \%)$. All 6 trials reported mortality data, and when the 6 trials were pooled in a meta-analysis, mortality at 1 year was not significantly different between IL-2based regimens and non-IL-2 regimens. These observations are consistent with those of Coppin and colleagues, ${ }^{6}$ who identified that objective response rate is not a particularly reliable surrogate for survival benefit in RCC. A sensitivity analysis showed that IL-2-based regimens, including 5-FU, were associated with a statistically significant reduction in 1-year mortality compared with non-IL-2 controls. However, features of these trials warrant that these immunochemotherapy regimens be further investigated in randomized trials before being considered standard treatment.

Two trials were conducted by the same
German cooperative group and were reported by the same principal investigator. The 2001 trial by Atzpodien and others, ${ }^{12}$ which showed the largest treatment effect, was a small trial $(n=78)$ and therefore at higher risk of false-positive results (type 1 error). Imbalances in important prognostic variables (i.e., age and possibly performance status) between trial arms might have influenced outcomes, resulting in a bias toward the IL-2 experimental arm; multivariate analyses adjusting for these were not reported. The 2004 trial by Atzpodien and others ${ }^{10}$ was better powered to detect differences between arms $(n=341)$; patient characteristics appeared well balanced, with the exception of a higher percentage of patients with metastases and patients who had not undergone nephrectomy in the control arm. However, only favourable risk patients were recruited into the trial, raising concerns about the generalizability of the results. Further limitations of the trial are discussed in an editorial by Negrier, ${ }^{19}$ which accompanies the published trial report. Negrier concluded that the trial findings should be interpreted with caution and validated in a rigorous controlled trial by others before being considered as reference treatment for future trials.

Overall, toxicity appeared consistently worse with IL-2-based therapy than with non-IL-2 therapy; however, most studies described IL-2 regimens as moderately to well tolerated by most patients. Specifically, hypotension, cardiac toxicity, diarrhea and fatigue were increased when compared with IFN-a or other treatment arms. No toxic deaths or quality-of-life data were reported in any of the trials. The general opinion is that IL-2-based regimens are associated with significant toxicity, and the magnitude of this toxicity may be underestimated in clinical trials because of patient selection factors. However, centres with expertise in high-dose IL-2 administration have developed approaches to manage and minimize these toxicities.

This review did not identify any ran- 
Hotte et al

domized trials comparing high-dose intravenous IL-2 to a non-IL-2 control or placebo; thus its true clinical effectiveness remains unclear. For this reason, it is impossible to recommend its use outside of clinical trials or investigative settings. A published series by Fisher and colleagues ${ }^{20}$ combining data $^{2}$ from 7 nonrandomized, single-arm phase II trials suggests that approximately $9 \%$ of patients can experience complete and long-lasting remissions with high-dose IL-2. The lack of control subjects makes it difficult to interpret these data. Patient selection would be expected with this relatively toxic therapy and could influence response and survival outcomes. For example, Motzer and others ${ }^{4}$ reported on a cohort of 670 RCC patients, of whom only $15 \%$ had prior IL-2-based therapy. In a favourable-risk group, identified by the absence of adverse clinical factors, the median survival was 20 months, similar to the median 16.3 months reported by Fisher and colleagues with high-dose IL-2. In addition, high-dose IL-2 was very resource intensive (most required intensive care unit admission for administration). It remains unclear whether the small proportion of patients who experience long-lasting remissions with highdose IL-2 are truly benefiting from this therapy or whether they are simply those with a superb prognosis identified within a population with an excellent prognosis by response to IL-2 therapy. Only controlled clinical trials can definitively answer this question.

Randomized trials of IFN-a-based immunotherapy, as well as cytoreductive nephrectomy combined with IFN-a, have shown modest survival benefits in this patient population and should therefore be considered as treatment options. Despite many years of research, the prognosis for patients with inoperable, locally advanced or metastatic RCC had not changed until recently, and very few therapeutic options existed for these patients; however, preliminary evidence from randomized trials of angiogenesis inhibitors, namely sunitinib, ${ }^{21-23}$ sorafenib ${ }^{24,25}$ and temsirolimus, ${ }^{26,27}$ are showing promising positive results with acceptable toxicity. The clinical benefits observed with angiogenesis inhibitors in these patients are a major step toward improving their poor prognosis. In Canada, most physicians consider IFN-a the standard of care for this patient population; however, recent results from randomized trials evaluating inhibitors of angiogenesis show superior clinical benefits over IFNa-based immunotherapy (and placebo) with an acceptable toxicity profile, making these agents preferred treatment options. The use of these agents will likely supplant IL-2 and IFN-a therapy as the preferred treatment modality in patients with advanced RCC. Notably, because not all patients will have access to the newer angiogenesis therapies due to funding constraints, information concerning IL-2 and IFN-a is still timely for some patients. Regardless, patients with inoperable, locally advanced or meta-static RCC continue to have an incurable malignancy, making further research necessary.

\section{Conclusions}

In patients with unresectable or metastatic RCC, immunotherapy with IL-2 adds toxicity but does not provide superior treatment efficacy over non-IL-2 regimens. There is some evidence that IL-2 combined with IFN-a and 5-FU may improve response rates and survival when compared with either agent alone or with a nonimmunotherapy control. However, those findings require confirmation in properly powered clinical trials with appropriate comparators (i.e., IFN-a) and should not be considered a standard of care. There are insufficient data to support the routine use of high-dose intravenous IL-2 therapy outside of a clinical trial or investigative setting, and its unique toxicity warrants its administration in specialized centres equipped to deal with specific toxicities and provide comprehensive care. 


\section{Clinical Practice Guideline}

The GU DSG deliberated all comments provided by the external review process but decided not to make any substantive modifications to the practice guideline. More detailed survey results and the GU DSG's specific responses to the written comments are available at www.cancercare.on.ca.

\section{Target population}

These recommendations apply to adult patients with unresectable or metastatic RCC.

\section{Recommendations}

Regimens containing non-high-dose IL-2 should not be used as standard treatment for unresectable or metastatic RCC.

High-dose IL-2 should only be used by experienced physicians in the context of a clinical trial or investigative setting.

\section{Qualifying statements}

Patients with unresectable or metastatic RCC should be encouraged to participate in clinical trials.

IFN-a and IFN-a combined with cytoreductive nephrectomy show modest survival benefits in randomized trials and may be considered treatment options in this patient population.

Preliminary evidence from randomized trials of inhibitors of angiogenesis (i.e., sunitinib, sorafenib and temsirolimus) show promising positive results with acceptable toxicity. These agents will likely supplant IFN-a as the preferred treatment modality in this patient population.

High-dose IL-2 has not been compared with appropriate comparators, using nonIL-2-containing regimens in randomized trials; thus its effectiveness is unclear. Despite this, high-dose IL-2 is being used as a standard treatment for unresectable or metastatic RCC in much of the US and parts of Europe, based on single-arm studies.
From the *Juravinski Cancer Centre and the ${ }^{\dagger}$ Cancer Care Ontario Program in Evidence-based Care, McMaster University, Hamilton, Ont., the $¥$ Ottawa Hospital Regional Cancer Centre Ottawa, Ont., and the $\S$ London Health Sciences Centre, London, Ont.

Acknowledgements: This systematic review and practice guideline were produced by the Program in Evidence-based Care (www.cancercare .on.ca). The Program is sponsored by Cancer Care Ontario and the Ontario Ministry of Health and Long-Term Care but is editorially independent.

This article has been peer reviewed.

Competing interests: None declared.

References

1. Greenlee RT, Murray T, Bolden S, et al. Cancer statistics 2000. CA Cancer I Clin 2000:50:7-33.

2. Canadian Cancer Society/National Cancer Institute of Canada: Canadian Cancer Statistics. Toronto: National Cancer Institute of Canada; 2006.

3. Bukowski RM. Immunotherapy in renal cell carcinoma. Oncology 1999; 13:801-10.

4. Motzer RJ, Mazumdar M, Bacik J, et al. Survival and prognostic stratification of 670 patients with advanced renal cell carcinoma. J Clin Oncol 1999; 17:2530-40.

5. Amato RJ. Chemotherapy for renal cell carcinoma. Semin Oncol 2000; 27:177-86.

6. Coppin C, Porzsolt F, Kumpf J, et al. Immunotherapy for advanced renal cell cancer. Cochrane Database Syst Rev 2004;3:CD001425.

7. Lipsey MW, Wilson DB. Practical meta-analysis. Thousand Oaks: Sage Publications; 2001

8. DerSimonian R, Laird N. Meta-analysis in clinical trials. Control Clin Trials 1986;7(3):177-88.

9. Higgins JPT, Thompson SG, Deeks JJ, et al. Measuring inconsistency in meta-analyses. BMJ 2003;327:557-60.

10. Atzpodien J, Kirchner $\mathrm{H}$, Jonas $\mathrm{U}$, et al. Interleukin-2 and interferon alfa2a-based immunochemotherapy in advanced renal cell carcinoma: a prospectively randomized trial of the German Cooperative Renal Carcinoma Chemoimmunotherapy Group (DGCIN). J Clin Oncol 2004;22:1188-94.

11. Brinkmann OA, Hertle L. Kombinierte Zytokin-therapie vs. misteltherapie bei metastasiertem nierenzellkarzinom. Klinischer vergleich des therapieerfolgs einer kombinierten gabe von interferon alfa-2b, interleukin-2 und 5-fluorouracil gegenüber einer behandlung mit mistellektin [German] der Onkologe 2004;10:978-85.

12. Atzpodien J, Kirchner H, lliger HJ, et al. IL-2 in combination with INF-a and 5 -FU versus tamoxifen in metastatic renal cell carcinoma: long term results of a controlled clinical trial. Br J Cancer 2001;85:1130-6.

13. Boccardo F, Rubagotti A, Canobbio L, et al. Interleukin-2, interferon-a and interleukin-2 plus interferon-a in renal cell carcinoma. A randomized phase II trial. Tumori 1998;84:534-9.

14. Henriksson R, Nilsson $S$, Colleen $S$, et al. Surivival in renal cell carcinoma-a randomized evaluation of tamoxifen vs interleukin-2, alphainterferon (leucocyte) and tamoxifen. Br J Cancer 1998;77:1311-7.

15. Negrier S, Escudier B, Lasset C, et al. Recombinant human interleukin2 , recombinant human interferon alfa-2a, or both in metastatic renalcell carcinoma. Groupe Français d'Immunothérapie. N Engl J Med 1998; 338:1272-8.

16. Lummen $G$, Goepel M, Mollhoff S, et al. Phase II study of interferongamma versus interleukin-2 plus interferon-alpha $2 \mathrm{~b}$ in metastatic renal cell carcinoma. J Urol 1996; 155:455-8. 
17. Baaten $G$, Voogd AC, Wagstaff J. A systematic review of the relation between interleukin-2 schedule and outcome in patients with metastatic renal cell cancer. Eur I Cancer 2004;40:1127-44.

18. Malaguarnera M, Ferlito L, Gulizia G, et al. Use of interleukin-2 in advanced renal carcinoma: meta-analysis and review of the literature. Eur I Clin Pharmacol 2001;57:267-73.

19. Negrier S. Better survival with interleukin-2-based regimens? Possibly only in highly selected patients. J Clin Oncol 2004;22:1174-6.

20. Fisher RI, Rosenberg SA, Fyfe G. Long-term update for high-dose recombinant interleukin-2 in patients with renal cell carcinoma. Cancer J Sci Am 2000;6(Suppl 1):S55-S57.

21. Motzer R, Hutson T, Tomczak P, et al. Phase 3 randomized trial of sunitinib malate (SU1 1248) versus interferon-alfa as first-line systemic therapy for patients with metastatic renal cell carcinoma (mRCC). [abstract] [Ann Meet Proc]. J Clin Oncol 2006;24(Suppl 18):LBA3.

22. Motzer R, Hutson T, Tomczak $P$, et al. Phase III randomized trial of sunitinib malate (SU11248) versus interferon-alpha (IFN-a) as firstline systemic therapy for patients with metastatic renal cell carcinoma (mRCC). [slides online]. Available: Www.asco.org/portal/site/ASCO /menuitem.34d60f5624ba07fd506fe310ee37a01d/?vgnextoid $=76 f 8201$ eb61a7010VgnVCM100000ed730ad I RCRD\&vmview=abst detail_view\&conflD=40\&abstract|D=30512. Accessed 2006 Sept 1.

23. Motzer RJ, Hutson TE, Tomczak P, et al. Sunitinib versus interferon alfa in metastatic renal-cell carcinoma. N Engl J Med 2007;356:115-24.
24. Escudier B, Szczylik C, Demkow T, et al. Randomized phase II trial of the multi-kinase inhibitor sorafenib versus interferon (IFN) in treatment-naive patients with metastatic renal cell carcinoma (mRCC). [abstract] [Ann Meet Proc] J Clin Oncol 2006;24(Suppl 18):4501.

25. Escudier B, Eisen T, Stadler W, et al. Sorafenib in advanced clear-cell renal-cell carcinoma. N Engl J Med 2007;356:125-34.

26. Hudes $G$, Carducci $M$, Tomczak $P$, et al. A phase 3, randomized 3arm study of temsirolimus (TEMSR) or interferon-alpha (IFN) or the combination of TEMSR + IFN in the treatment of first-line, poor-risk patients with advanced renal cell carcinoma (adv RCC). [abstract] [Ann Meet Proc] I Clin Oncol 2006;24(Suppl 18):LBA4.

27. Hudes $G$, Carducci M, Tomczak $P$, et al. A phase 3, randomized, 3-arm study of temsirolimus (TEMSR) or interferon-alpha (IFN) or the combination of TEMSR + IFN in the treatment of first-line, poor-risk patients with advanced renal cell carcinoma. [slides online]. Available: www.asco.org /portal/site/ASCO/menuitem.34d60f5624ba07fd506fe310ee37a0ld /?vgnextoid=76f8201eb6la7010VgnVCM100000ed730ad IRCRD\&umview $=$ abst_detail_view\&conflD=40\&abstract|D=30421. Accessed 2006 Sept 1 .

Correspondence: Dr. Sebastien Hotte, Juravinski Cancer Centre, 699 Concession Street, Level 3, Hamilton ON L8V 5C2; Sebastien.Hotte@hrcc.on.ca
We accept events for the Calendar section. You may send them to us in the following ways:

\section{E-mail \\ journal@cua.org}

Fax

514 875-0205
Mail
CUAJ
1155 University Ave., Suite 1014
Montreal, QC H3B 3A7

\section{First International Workshop on Focal Therapy and Imaging of Prostate Cancer}

\section{February 22-23, 2008}

Duke University Medical Center, Durham, NC, USA

Contact person: Mrs. Rae Sidlauskas

E-mail: rachel.sidlauskas@duke.edu

Phone: 919 684-2033

Fax: 919 684-4611

Web site: urology.surgery.duke.edu 